\title{
Benefícios de um programa de atividade física para a melhoria da qualidade de vida de idosos no estado do Rio de Janeiro
}

\author{
Maria Angélica Sanchez ${ }^{\star}$, Joice Mariana Moraes Brasil**, Izabel Aparecida Mendonça Ferreira***
}

\section{Resumo}

O presente trabalho traz os resultados dos benefícios de um programa de atividade física na qualidade de vida de idosos no estado do Rio de Janeiro. O programa, implantado no ano de 2013, é composto por uma equipe multidisciplinar e atende indivíduos de 45 municípios do estado. Para aferir a qualidade de vida, o questionário SF 36, versão de onze itens, validado para uso no Brasil, foi aplicado no momento de ingresso no programa. Após oito meses frequentando o programa, todos os indivíduos, que compareceram às atividades por, pelo menos, três vezes por semana, foram submetidos a uma nova avaliação pela escala SF 36, sendo selecionados para análise todos aqueles com 60 anos ou mais. Dentre os 4.032 indivíduos que ingressaram no programa, 402 idosos desse estrato etário, distribuídos por 28 municípios, responderam à escala pela segunda vez. A média de idade dos participantes foi de $67,9( \pm 6,3)$ anos. Os resultados mostram que houve alteração em todos os domínios, sinalizando para a melhora da capacidade funcional e para o melhor desempenho nos demais do- mínios mensurados pela escala, sugerindo que a prática de exercícios físicos exerce efeito positivo sobre a qualidade de vida da população que envelhece.

Palavras-chave: Idosos. Atividade física. Qualidade de vida.

\section{Introdução}

Questões referentes ao processo de envelhecimento populacional, tais como as morbidades, as políticas públicas, os novos paradigmas de atenção ao idoso, tornaram-se alvo de discussões e estudos. As novas demandas desse processo trazem a necessidade da readequação das ações voltadas para esse segmento da população (CAMARANO, 2014).

Em decorrência do envelhecimento humano, nas últimas décadas, os perfis de saúde da população também passaram por transformações, mais evidentes com o aparecimento das doenças crônico-

* Doutora em Ciências. Pesquisadora do Instituto de Desenvolvimento Institucional e Ação Social (Ideias). Universidade Estadual do Rio de Janeiro (Uerj). Endereço para correspondência: Policlínica Piquet Carneiro / Uerj Av. Mal Rondon 381, 2º andar. São Francisco Xavier, Rio de Janeiro - RJ CEP 20950-003. E-mail: asanchezrj@gmail.com

** Especialista em Geriatria e Gerontologia. Pesquisadora Júnior do Instituto de Desenvolvimento Institucional e Ação Social (Ideias). E-mail: joicebrasil30@gmail.com

**** Assessora técnica na área de projetos do Instituto de Desenvolvimento Institucional e Ação Social (Ideias). E-mail: assessoria.ideiasrj@gmail.com

$\rightarrow$ http://dx.doi.org/10.5335/rbceh.2014.4528

Recebido em: 01/12/2014. Aceito em: 06/05/2015 
-degenerativas, que elevam o número de pessoas idosas susceptíveis a doenças cardiovasculares, artrites, fraturas e vulneráveis a uma série de outras situações adversas (CHAIMOWICZ; CAMARGOS, 2011). Entre essas situações, destaca-se o desenvolvimento da dependência, a ocorrência de quedas e lesões, o surgimento de doenças agudas, a institucionalização e lentidão na recuperação da saúde (LOURENÇO, 2008).

Diante das condições adversas, que em parte se associam ao processo de envelhecimento e apresentam alta prevalência na população idosa, a prevenção é a ação de maior relevância e a atividade física parece colaborar sobremaneira para a diminuição do risco de desenvolvimento de várias doenças (MACIEL et al., 2013; SILVA et al., 2014; SIQUEIRA et al., 2008;).

A despeito dos efeitos positivos que a atividade física pode proporcionar a qualquer indivíduo, sua prática é ainda pouco valorizada. Em um estudo nas Regiões Sul e Nordeste do Brasil, em uma amostra composta por 4.003 idosos, com o objetivo de descrever a prevalência de sedentarismo e fatores associados, os autores estimaram o predomínio de $58 \%$ de sedentarismo na população com 60 anos ou mais, refletindo a necessidade de maiores investimentos em ações dessa natureza (SIQUEIRA et al., 2008).

A prática regular de exercício físico parece ser uma das melhores iniciativas para a melhoria da qualidade de vida, ao passo que um estilo de vida sedentário pode representar um fator de risco para o surgimento de doenças crônico-degenerativas (MACIEL et al.,
2013). Em estudo realizado no estado do Rio Grande do Sul, com uma amostra composta por 863 participantes, jovens e adultos, a prática de atividade física mostrou-se como mola propulsora na melhora da qualidade de vida em vários aspectos. Os autores observaram que quanto mais ativo é o indivíduo, melhor é sua qualidade de vida, incluindo não apenas as questões relacionadas à saúde, mas também aos aspectos psicológicos e cognitivos (SILVA et al., 2010).

O termo qualidade de vida tem sido amplamente utilizado em diversas áreas para denominar uma situação que se aproxime de uma satisfação integral, seja na família, nas relações afetivas, na vida social e ambiental, determinando o padrão de conforto e bem-estar (MINAYO; HARTZ; BUSS, 2000). Trata-se de conceito ainda subjetivo, porém, está diretamente ligado ao processo multifatorial que influencia o bem-estar físico, psicossocial e econômico. É dependente do meio sociocultural e das aspirações pessoais. Está relacionado à autoestima abrangendo aspectos significativos que impactam nas habilidades funcionais e fisiológicas (GAULT; WILLEMS, 2013).

Em face de sua natureza abstrata, o termo qualidade de vida pode variar conforme a cultura, a classe social e a época em que se está vivendo. Indo além, pode variar de indivíduo para indivíduo. Importante, porém, é que tal termo tem múltiplas dimensões, como a física, a social e a psicológica (PASCHOAL, 2011).

No que tange ao processo de envelhecimento, alguns declínios substanciais se instalam ao longo dos anos e podem ocasionar a diminuição das reservas 
fisiológicas e, consequentemente, o aumento de marcadores biológicos, produzindo alterações intrínsecas (sistema cardiovascular, metabólica, condução fibra/muscular) e extrínsecas (alteração no sistema musculoesquelético, declínio na capacidade funcional, síndrome de imobilidade), desencadeando a vulnerabilidade do indivíduo e incidindo em mudanças substanciais na qualidade de vida (GAULT; WILLEMS, 2013).

Dentre os instrumentos utilizados para mensurar a qualidade de vida, destaca-se a escala SF 36 (WARE; SHERBOURN, 1992), que em uma de suas versões reduzidas apresenta-se como uma escala de onze itens para avaliar oito dimensões de saúde: capacidade funcional, aspecto físico, dor, estado geral de saúde, vitalidade, aspectos sociais, aspectos emocionais e saúde mental.

A relação entre a tríade atividade física, saúde, qualidade de vida é cada vez mais analisada cientificamente quando se discute o processo de envelhecimento. Parece consenso entre os profissionais da área da saúde que a atividade física é um dos fatores determinantes para o processo de envelhecer com saúde (MATSUDO, S. M.; MATSUDO, V. K. R.; BARROS NETO, 2001; SILVA et al., 2010; PUCCI et al., 2012; GAULT; WILLEMS, 2013), e que a qualidade de vida como um determinante multifacetado pode ser mensurada a partir de outras ações que promovam o bem-estar de forma abrangente. Diante desse cenário, o retrato ideal é aquele em que as políticas públicas possam estar voltadas para 0 aspecto preventivo e de estímulo às ações destinadas a melhorar as condições de vida e minimizar os efeitos adversos produzidos pela precariedade do estilo de vida atualmente praticado.

O Brasil possui um cabedal de marcos legais totalmente direcionado à implementação de ações que possam ser carreadoras de um envelhecimento bem-sucedido. Por exemplo, o estímulo à prática de atividades físicas, que é tópico do Estatuto do Idoso (BRASIL, 2003) e da Política Nacional de Saúde da Pessoa Idosa (BRASIL, 2006), recentemente, entrou no rol de ações governamentais. Em vários estados do país, sobretudo nas regiões Sul e Sudeste, projetos de academias da terceira idade são implementados. No entanto, há que se avaliar a eficácia das práticas de modo a aprimorar suas estratégias.

O presente estudo tem como objetivo analisar as mudanças na qualidade de vida dos idosos inseridos em um programa de promoção de saúde a partir da análise das avaliações realizadas. ${ }^{1}$

\section{Método}

Quanto ao delineamento e aos participantes, este estudo é transversal e observacional, utilizando dados secundários obtidos no cadastro dos idosos que ingressaram em um programa de atividade física.

O programa consiste em desenvolver em municípios do estado do Rio de Janeiro atividades coordenadas por uma equipe multidisciplinar composta por um profissional de educação física, responsável pelas atividades executadas nos equipamentos implantados nas praças das cidades, um fisioterapeuta, 
que realiza as sessões de alongamento e exercícios de propriocepção após as atividades, e um nutricionista, que fornece orientações acerca de estilo de vida saudável e educação alimentar.

A população do estudo foi formada por 4.032 indivíduos com idade igual ou superior a 45 anos, inseridos no programa de atividade física nas academias da terceira idade. Dentre esses, 2.261 residentes em 28 municípios do estado do Rio de Janeiro (Quadro 1) foram reavaliados. Foram analisados os dados de todos aqueles com 60 anos ou mais que responderam à segunda aplicação do questionário de avaliação, perfazendo um total de 402 idosos.

$\mathrm{O}$ instrumento utilizado para analisar o impacto da atividade física foi o Medical Outcomes Study 36-Item Short-Form Health Survey (SF-36), um questionário genérico que avalia aspectos da qualidade de vida que estão diretamente relacionados à saúde do indivíduo. Para o ingresso no programa, optou-se pela entrevista individual, utilizando a versão reduzida, composta por onze itens e validada para uso no Brasil (CICONELLI et al., 1997). Todos os idosos foram submetidos a um protocolo que contou com instrumentos de avaliação da qualidade de vida, capacidade funcional e avaliação nutricional.
Quadro 1:Municípios por número e percentual de indivíduos que foram submetidos à reavaliação com a reaplicação da escala SF $36(\mathrm{n}=2.261)$

\begin{tabular}{|c|c|c|}
\hline Município & $\mathrm{n}$ & $\%$ \\
\hline Areal & 94 & 4,1 \\
\hline Belford Roxo & 54 & 2,4 \\
\hline Bom Jardim & 81 & 3,6 \\
\hline Bom Jesus de Itabapoana & 73 & 3,2 \\
\hline Búzios & 78 & 3,4 \\
\hline Cambuci & 110 & 4,9 \\
\hline Carmo & 61 & 2,6 \\
\hline Duque de Caxias & 5 & 0,2 \\
\hline Itaipava & 130 & 5,7 \\
\hline Italva & 63 & 2,8 \\
\hline Japeri & 131 & 5,8 \\
\hline Miguel Pereira & 32 & 1,4 \\
\hline Nilópolis & 28 & 1,2 \\
\hline Nova Iguaçu & 51 & 2,3 \\
\hline Paracambi & 9 & 0,4 \\
\hline Paraíba do Sul & 112 & 5,3 \\
\hline Petrópolis & 177 & 7,7 \\
\hline Queimados & 141 & 5,9 \\
\hline Resende & 98 & 4,2 \\
\hline São Francisco de Itabapoana & 66 & 2,8 \\
\hline São Pedro da Aldeia & 27 & 1,1 \\
\hline Sapucaia & 32 & 1,4 \\
\hline Saquarema & 96 & 4,2 \\
\hline Sumidouro & 26 & 1,1 \\
\hline Tanguá & 164 & 7,2 \\
\hline Três Rios & 32 & 1,4 \\
\hline Volta Redonda & 252 & 12,0 \\
\hline Valença & 38 & 1,7 \\
\hline Total & 2.261 & 100 \\
\hline
\end{tabular}

Fonte: dados da pesquisa. 
A SF 36 foi aplicada pelo profissional de educação física, responsável pelas atividades em cada município. As entrevistas foram registradas em formulário próprio e, posteriormente, armazenadas no sistema de informações do programa. Todos os indivíduos que ingressaram no programa foram submetidos à aplicação da SF 36,-instrumento que fazia parte do protocolo de avaliação. A aplicação da escala durava em média 10 minutos, e todos aqueles que aderiram ao programa e frequentaram as atividades, por pelo menos três vezes por semana, foram submetidos à segunda aplicação do questionário após oito meses de inserção nas atividades.

Os dados foram armazenados no sistema de informação da secretaria de estado e importados para análise no software SPSS 19.0. Foram avaliadas as frequências descritivas para os dados sociodemográficos $\mathrm{O}$ desempenho dos indivíduos, após oito meses de prática de exercício, foi comparado por meio do teste t de student pareado $(\mathrm{p}<0,001)$.

\section{Resultados}

Um total de 4.032 indivíduos ingressou no programa de atividade física entre outubro de 2013 e junho de 2014. Dentre esses, 402 indivíduos com 60 anos ou mais, distribuídos em 28 municípios, foram submetidos à reaplicação da SF 36. A média de idade dos participantes foi de 67,9 $( \pm 6,3)$ anos (Tabela 1).

Após oito meses de atividades, foram observadas mudanças em todos os domínios, sinalizando para a melhora da capacidade funcional e melhor do desempenho nos demais domínios mensurados pela escala (Tabela 2 ).

Tabela 1: Sexo, idade e escolaridade dos idosos que foram submetidos à reaplicação da SF 36(n=392)

\begin{tabular}{l|r}
\hline \multicolumn{1}{c|}{ Características } & \multicolumn{1}{c}{$\mathrm{n}(\%)$} \\
\hline Sexo & $78(19,9)$ \\
Masculino & $314(80,1)$ \\
Feminino & \\
Idade & $147(37,5)$ \\
60 a 64 anos & $104(26,5)$ \\
65 a 69 anos & $77(19,6)$ \\
70 a 74 anos & $38(9,7)$ \\
75 a 79 anos & $26(6,6)$ \\
80 anos ou mais & \\
Escolaridade & $370(94,4)$ \\
Ensino fundamental & $17(4,3)$ \\
Ensino médio & $5(1,3)$ \\
Ensino superior &
\end{tabular}

Fonte: dados da pesquisa. 
Tabela 2: Comparação entre os oito domínios da SF 36 - oito meses após ingresso no programa de atividade física $(n=402)$, utilizando o teste t pareado

\begin{tabular}{|c|c|c|c|}
\hline Domínios & Médias & Diferença das médias (IC) & $\mathrm{P}$ \\
\hline \multicolumn{4}{|l|}{ Capacidade funcional } \\
\hline 1a aplicação & 62,915 & $72,500(70,234-74,765)$ &, 000 \\
\hline $2^{a}$ aplicação & 70,670 & $80,509(78,523-82,496)$ &, 000 \\
\hline \multicolumn{4}{|l|}{ Aspectos físicos } \\
\hline $1^{\text {a }}$ aplicação & 30,788 & $66,044(61,824-70.264)$ &, 000 \\
\hline $2^{\underline{a}}$ aplicação & 44,617 & $80,099(76,570-83,628)$ &, 000 \\
\hline \multicolumn{4}{|l|}{ Dor } \\
\hline 1a aplicação & 68,818 & $65,131(62,584-67,678)$ &, 000 \\
\hline $2^{\underline{a}}$ aplicação & 50,274 & $78,171(75,938-80,404)$ &, 000 \\
\hline \multicolumn{4}{|l|}{ Estado geral de saúde } \\
\hline 1a aplicação & 77,783 & $66,097(64,426-67,767)$ &, 000 \\
\hline $2^{a}$ aplicação & 77,784 & $68,858(67,122-70,594)$ &, 000 \\
\hline \multicolumn{4}{|l|}{ Vitalidade } \\
\hline 1a aplicação & 66,944 & $71,281(69,187-73,374)$ &, 000 \\
\hline 2ª aplicação & 88,885 & $79,751(77,987-81,515)$ &, 000 \\
\hline \multicolumn{4}{|l|}{ Aspectos sociais } \\
\hline $1^{a}$ aplicação & 61,996 & $79,322(76,806-81,837)$ &, 000 \\
\hline $2^{a}$ aplicação & 93,364 & $89,552(87,666-91,437)$ &, 000 \\
\hline \multicolumn{4}{|l|}{ Aspectos emocionais } \\
\hline 1aㅡ aplicação & 29,966 & $68,739(64,229-73,249)$ &, 000 \\
\hline $2^{\underline{a}}$ aplicação & 51,667 & $84,494(81,279-87,709)$ &, 000 \\
\hline \multicolumn{4}{|l|}{ Saúde mental } \\
\hline 1a aplicação & 74,302 & $76,318(74,299-78,337)$ &, 000 \\
\hline $2^{a}$ aplicação & 88,760 & $81,273(79,473-83,073)$ & ,000 \\
\hline
\end{tabular}

Fonte: dados da pesquisa.

Nota: $1^{\mathrm{a}}$ aplicação - Antes de iniciar as atividades; $2^{\mathrm{a}}$ aplicação - Oito meses após o início das atividades; $\mathrm{P}<0,001$. 


\section{Discussão}

O presente estudo teve como finalidade analisar se a prática de atividade física regular, desenvolvida por uma equipe multidisciplinar, mostraria algum benefício na qualidade de vida dos idosos. Para essa análise, foi utilizada a escala de qualidade de vida (SF 36) que apresentou um retrato do indivíduo antes e depois da inserção em um programa de promoção de saúde. Foram observadas mudanças de comportamento em todos os domínios da escala utilizada, indicando que a prática de exercícios físicos pode ser um preditor de melhoria na qualidade de vida.

Como na maioria das atividades de convivência, a predominância das mulheres foi observada. Em estudo realizado na Escola de Educação Física da USP, em 2009, os autores obtiveram a participação de $74 \%$ de mulheres (PEREIRA; OKUMA, 2009), percentual semelhante ao encontrado nesta pesquisa. Esse fato pode estar associado ao maior número de mulheres na população brasileira. Aliado a isso, cabe ressaltar que as mulheres, geralmente aderem com mais facilidade aos programas de atividade física e dão mais importância às atividades que requerem a interação com grupos (GOGGIO; MORROW JUNIOR, 2001).

Um aspecto fundamental para a realização de atividades físicas é a manutenção da capacidade funcional das pessoas que envelhecem, principal finalidade da Política Nacional de Saúde da Pessoa Idosa, que apresenta como uma de suas diretrizes a promoção do envelhecimento ativo e saudável (BRA-
SIL, 2006). Em estudo realizado com um grupo de 94 idosos, integrantes de um programa de atividade física em Rio Claro (SP), com o objetivo de analisar o efeito de três modalidades de exercícios físicos na capacidade funcional desses indivíduos, evidenciou-se que a prática de atividade física orientada sistematizada é uma ação eficaz para a manutenção de componentes de capacidade funcional, independente da modalidade das atividades implementadas (UENO et al., 2012). No presente estudo foi, igualmente, observada a melhora da capacidade funcional dos envolvidos no programa.

No domínio de aspectos físicos, quando analisados os dados, observou-se que o desempenho nos aspectos físicos foi um dos domínios que melhor se modificou após a inserção dos idosos no programa. Tal alteração de comportamento pode ser atribuída à prática regular de exercícios. A inatividade física pode ser causa primária para o baixo desempenho nas atividades de vida diária. No entanto, a inserção em programas de atividades físicas pode promover mudanças qualitativas no estilo de vida dos indivíduos que as realizam (FRANCHI et al., 2008).

Os indivíduos envolvidos no presente estudo também relataram melhoras nos quadros álgicos, demonstrando que a inserção no programa foi capaz de diminuir dores. Em estudo de revisão, foram encontrados vários aspectos positivos nos trabalhos em que exercícios físicos e tratamentos multidisciplinares substituíam o tratamento convencional, ou seja, repouso e fármacos (SOUZA, 2009).

No que tange ao estado geral de saúde, a resposta da inserção no progra- 
ma constituiu um aspecto positivo. Já se discute amplamente sobre os efeitos benéficos do engajamento em atividades físicas. Em estudo realizado na cidade de São José (SC), com 242 idosos, os autores concluíram que a atividade física exerce um efeito benéfico nas condições de saúde, além de reduzir a incidência de quedas na população (MAZO et al., 2007).

Quanto aos aspectos sociais, emocionais e de saúde mental, muitos estudos já discorreram sobre os benefícios da atividade física. $\mathrm{Na}$ amostra desta pesquisa, os resultados não foram diferentes. Além da interação social, que se mostrou como um indicador de adesão ao programa, as análises mostraram alterações significativas nos três domínios. No estudo sobre a dimensão psicossocial da atividade física na velhice, realizado em Natal (RN), abordando as representações sociais, concluiu-se que idosos participantes de um grupo de atividades compartilham representações consensuais sobre a importância da atividade física, o que permitiu para alguns ressignificar o conceito de atividade física, incorporando-lhe novos significados, como bem-estar, disposição e amizade (SILVA, 2011).

\section{Conclusões}

Os resultados do presente trabalho permitem inferir que a prática de exercícios físicos regulares e o aporte de profissionais de fisioterapia e nutrição em atividades complementares voltadas para a adoção de hábitos saudáveis são medidas eficazes para se alcançar um envelhecimento com melhor qualidade de vida.

O presente estudo corrobora achados prévios que relatam os benefícios da atividade física para a população que envelhece. $\mathrm{O}$ arsenal brasileiro de políticas públicas é vasto e sempre acena para a promoção de saúde, no entanto é necessário a implementação desses procedimentos e, ainda, a viabilização do acesso da população interessada. $\mathrm{O}$ estímulo às atividades que preservem a capacidade funcional deve ser alvo das ações propostas tanto na esfera pública quanto na privada.

\section{Benefits of a physical activity program to improve the quality of life of elderly in the state of Rio de Janeiro}

\section{Abstract}

This paper presents the results of the impact of a physical activity program on quality of life of elderly people in the state of Rio de Janeiro. The program, established in 2013, is composed of a multidisciplinary team and includes individuals from about 45 municipalities in the state. To assessment the quality of life SF 36, 11-item version, validated for use in Brazil, was applied at the time of joining the program. After eight months, all individuals aged 60 or older who have joined the project and attended the activities for, at least three times a week, were invited to the reapplication of scale. Of the 4,032 individuals who entered the program, 402 elderly people spread over 28 municipalities, were submitted to a second administration of the scale. The average age of participants was 67.9 $( \pm 6.3)$ years. The results shows that there were changes in all dimensions of the scale. It shows the improvement in functional capacity and performance, suggesting that physical exercise has a positive effect on the quality of life of the aging population.

Keywords: Elderly. Physical activity. Quality of life. 


\section{Nota}

1 Trata-se de análise de dados secundários em que foram analisados os cadastros dos idosos inseridos no programa. Todos foram consultados e informados de que nenhuma informação que os identificassem seria divulgada. Após esse processo, os participantes assinaram um termo de consentimento de uso desses dados em publicações. Os autores se responsabilizam pelas questões éticas e informam que a realização desse trabalho não incorreu em quaisquer transtornos físicos ou emocionais para os integrantes deste estudo.

\section{Referências}

BRASIL. Estatuto do idoso: Lei Federal no 10.741, de $1^{\circ}$ de outubro de 2003. Brasília. Disponível em: <http:/www.planalto.gov.br/ ccivil_03/leis/2003/110.741.htm>. Acesso em: 14 nov. 2014.

. Ministério da Saúde. Portaria no. 2.528, de 19 de outubro de 2006. Aprova a Política Nacional de Saúde da Pessoa Idosa e determina outras providências. Brasília, DF: Ministério da Saúde, 2006.

CICONELLI, R. M. et al. Tradução para a língua portuguesa e validação do questionário genérico de avaliação de qualidade de vida - SF-36 (Brasil SF 36). Revista Brasileira de Reumatologia, São Paulo, v. 39, n. 3, p. 143150, maio/jun. 1999.

CHAIMOWICZ, F; CAMARGOS, M. C. S. Envelhecimento e Saúde no Brasil. In: FREITAS, E. V.; PY, L. (Org.). Tratado de Geriatria e Gerontologia. Rio de Janeiro: Guanabara Koogan, 2011. p. 74-98.

CAMARANO, A. A. (Org.). Novo regime demográfico: uma nova relação entre população e desenvolvimento?. Rio de Janeiro: IPEA, 2014.

FRANCHI, K. M. B. et al. Capacidade funcional e atividade física de idosos com diabetes tipo 2. Revista Brasileira de Atividade Física \& Saúde, Pelotas, v. 13, n. 3, p. 158-166, 2008.
GOGGIO, N. L.; MORROW JUNIOR, J. R. Physical activity, beheaviors of older adults. Jornal of Aging and Physical Activity, Champaign-IL, v. 9, n. 1, p. 58-66, 2001.

GAULT, M. L.; WILLEMS, M. E. T. Aging, functional capacity and eccentric exercise training. Aging and Disease, Fort Worth-TX, v. 4, n. 6, p. 351-363, Sept. 2013.

LOURENÇO, R. A. A síndrome da fragilidade no idoso: marcadores clínicos e biológicos. Revista do Hospital Universitário Pedro Ernesto, Rio de Janeiro, v. 7, n. 1, p. 21-29, jan./jun. 2008.

MINAYO, M. C. S.; HARTZ, Z. M. A.; BUSS, P. M. Qualidade de vida e saúde: um debate necessário. Ciência \& saúde coletiva, Rio de Janeiro, v. 5, n. 1, p. 7-18, 2000.

MATSUDO, S. M.; MATSUDO, V. K. R.; BARROS NETO, T. L. Atividade física e envelhecimento: aspectos epidemiológicos. Revista Brasileira de Medicina do Esporte, Niterói, v. 7, n. 1, p. 2-13, 2001.

MAZO, G. Z. et al. Condições de saúde, incidência de quedas e nível de atividades física dos idosos. Revista Brasileira de Fisioterapia, São Carlos, v. 11, n. 6, p. 437-442, nov./dez. 2007.

MACIEL, E. S. et al. The relationship between physical aspects of quality of life and extreme levels of regular physical activity in adults. Cadernos de Saúde Pública, Rio de Janeiro, v. 29, n. 11, p. 2.251-2.260, nov. 2013.

PEREIRA, J. R. P.; OKUMA, S. Perfil dos ingressantes de um programa de educação física para idosos e os motivos da adesão inicial. Revista Brasileira de Educação Física e Esporte, São Paulo, v. 23, n. 4, p. 319-334, out./dez. 2009.

PASCHOAL, S. M. P. Qualidade de vida na velhice. In: FREITAS, E. V.; PY, L. (Org.). Tratado de Geriatria e Gerontologia. Rio de Janeiro: Guanabara Koogan, 2011. p. 74-98. 
PUCCI, G.C.M.F. et al. Associação entre atividade física e qualidade de vida em adultos. Revista de Saúde Pública, São Paulo, v. 46, n. 1, p. 166-179, fev. 2012.

SILVA, S. M. Dimensão psicossocial da atividade física na velhice. Fractal: Revista de Psicologia, Rio de Janeiro, v. 23, n. 2, p. 337-352, maio/ago. 2011.

SILVA, R. S. et al. Atividade física e qualidade de vida. Ciência \& Saúde Coletiva, Rio de Janeiro, v. 15, n. 1, p. 115-120, jan. 2010.

SILVA, P. A. B. et al. Ponto de corte para o WHOQOL-bref como preditor de qualidade de vida de idosos. Revista Saúde Pública, São Paulo, v. 48, n. 3, p. 390-397, jun. 2014.

SIQUEIRA, F. V. et al. Atividade física em adultos e idosos residentes em áreas de abrangência de unidades básicas de saúde de municípios das regiões Sul e Nordeste do Brasil. Cadernos de Saúde Pública, Rio de Janeiro, v. 24, n. 1, p. 39-54, jan. 2008.

SOUZA, J. B. Poderia a Atividade Física Induzir Analgesia em Pacientes com Dor Crônica? Revista Brasileira de Medicina do Esporte, Niterói, v. 15, n. 2, p. 145-150, mar./ abr. 2009.

UENO, D. T. et al. Efeitos de três modalidades de atividade física na capacidade funcional de idosos. Revista Brasileira de Educação Físicae Esporte, São Paulo, v. 26, n. 2, p. 273-281, abr.jun. 2012.

WARE, J. E.; SHERBOURNE C. D. The MOS 36 item short form health survey (SF36). I. Conceptual framework and item selection. Medical Care, v. 30, n. 6, p. 473-483, 1992. 